\title{
O pré-natal psicológico em gestação decorrente de estupro: protocolo de intervenção
}

\author{
Atención psicológica prenatal en casos de embarazo por violación: Protocolo de \\ intervención
}

\author{
Psychological prenatal in pregnancy due to rape: Intervention Protocol Prenatal \\ Psicológico
}

\author{
Elen Márcia Carioca Zerbini \\ Universidade de Brasília (UnB), Brasília - DF/Brasil \\ ORCID: 0000-0003-4366-2031 \\ E-mail: cariocaelen@gmail.com
}

\author{
Alessandra da Rocha Arrais \\ Universidade Católica de Brasília (UCB), Brasília - DF/Brasil \\ ORCID: 0000-0002-1057-6914 \\ E-mail: alearrais@gmail.com
}

\begin{abstract}
Resumo
Introdução: Quando uma mulher se descobre gestante em decorrência um crime de estupro é imprescindível que ela receba o cuidado em saúde adequado. O Pré-natal Psicológico um desses tipos de cuidado. Objetivo: Trata-se de uma proposta de intervenção relacionada à saúde mental no ciclo gravídico-puerperal, denominada pré-natal psicológico, que visa apresentar um modelo de trabalho para que as equipes de saúde, especialmente psicólogos perinatais, possam atuar nas dificuldades inerentes à gestação decorrente de estupro e minimizar os agravos psicológicos deste tipo de gestação. Método: Foi desenvolvida no Hospital Materno Infantil de Brasília (HMIB), como produto da dissertação "Contribuições do Prénatal Psicológico para proteção psíquica da díade mãe-bebê em caso de gestação decorrente de estupro: Um estudo de caso à luz da teoria da subjetividade". A pesquisa foi ancorada nos preceitos teórico-metodológicos qualitativos da Teoria da Subjetividade, e tratou-se de um estudo de caso de uma mulher de 29 anos, solteira, na $23^{\text {a }}$ semana de uma gestação decorrente de estupro, sobre a qual a gestante teve o seu pleito por aborto legal mal gerido, e foi obrigada a levar a gestação a termo. Ela iniciou um trabalho de Pré-Natal Psicológico (PNP) para que pudesse lidar com os agravos psíquicos do trauma, e com a escolha a ser feita após o nascimento do bebê, adoção ou tomá-lo como filho. Resultado: Foi possível sistematizar uma adaptação do modelo Pré-Natal Psicológico, baseado no acompanhamento psicológico realizado com a participante da pesquisa, que poderá ser utilizado por profissionais de saúde, especialmente psicólogos perinatais, que trabalhem com vítimas de violência sexual.
\end{abstract}

Palavras-chaves: Estupro; Gravidez; Cuidado pré-natal; Angústia psicológica; Intervenção.

\section{Resumen}

Introducción: Cuando una mujer se encuentra embarazada como consecuencia de uma violación, es fundamental que reciba una atención de salud adecuada. La atención prenatal psicológica es uno de estos tipos de atención. Objetivo: Se trata de una propuesta de intervención relacionada con la salud mental en el ciclo embarazo-puerperal, denominada atención psicológica prenatal, que tiene como objetivo presentar un modelo de trabajo para que los equipos de salud, especialmente los psicólogos perinatales, puedan actuar en las dificultades inherentes al embarazo derivadas de violación y minimizar su daño psicológico. Método: Fue desarrollado en el Hospital Materno Infantil de Brasília (HMIB), como producto de la disertación "Contribuciones de la atención Psicológica Prenatal para la protección psíquica de la díada madre-bebé en caso de embarazo producto de violación: un estudio de caso à la luz de la teoría de la subjetividad ". La investigación se basó en los preceptos teórico-metodológicos cualitativos de la Teoría de la Subjetividad, y se trató de un estudio de caso de una mujer soltera de 29 años, en la $23^{\circ}$ semana de embarazo producto de violación, sobre la cual la gestante tenía su reclamo por aborto legal mal 
administrado, y se vio obligada a llevar el embarazo a término. Inició un trabajo de Psicología Prenatal (PNP) para poder hacer frente al daño psicológico que le ocasionó el trauma, y con la elección a tomar después del nacimiento del bebé, adopción o tomarlo de niño. Resultados: Se logró sistematizar una adaptación del Modelo Psicológico de Prenatal, a partir del seguimiento psicológico realizado con el participante de la investigación, el cual puede ser utilizado por profesionales de la salud, especialmente psicólogos perinatales, que trabajan con víctimas de violência sexual.

Palabras clave: Violación; Embarazo; Cuidado prenatal; Trastorno psicológico; Intervención.

\begin{abstract}
Introduction: When a woman finds herself pregnant due to a crime of rape, it is essential that she receives adequate health care. Psychological prenatal care is one of these types of care. Objective: This is a proposal for intervention related to mental health in the pregnancypuerperal cycle, called psychological prenatal care, which aims to present a working model so that health teams, especially perinatal psychologists, can act in the difficulties inherent to pregnancy resulting from rape and
\end{abstract}

minimizing the psychological harm of this type of pregnancy. Method: It was developed at the Hospital Materno Infantil de Brasília (HMIB), as a product of the masters dissertation "Contributions of Psychological Prenatal care for the psychic protection of the motherinfant dyad in case of pregnancy resulting from rape: A case study in the light of the theory of subjectivity". The research was based on the qualitative theoreticalmethodological precepts of the Theory of Subjectivity, and it was a case study of a 29-year-old single woman, in the 23 rd week of pregnancy resulting from rape, about which the pregnant woman had the her claim for legal abortion mismanaged, and was forced to carry the pregnancy to term. She started the treatment of Psychological Prenatal (PNP) so that she could deal with the psychological damage caused by trauma, and with the choice to be made after the baby's birth, adoption or taking it as a child. Results: It was possible to systematize an adaptation of the Psychological Prenatal Model, based on the psychological follow-up carried out with the research participant, which can be used by health professionals, especially perinatal psychologists, who work with victims of sexual violence.

Keywords: Rape; Pregnancy; Prenatal Care; Psychological distress; Intervention.

\section{Introdução}

No Distrito Federal, o Programa de Interrupção Gestacional Prevista em Lei (PIGL) da Secretaria de Estado de Saúde, é o programa que atende mulheres que pleiteiam o direito de interromper a gravidez em um dos casos previstos em lei: gravidez em decorrência de estupro. Desde 1996, através da resolução ${ }^{\circ}$ 1 do Conselho de Saúde do DF, o Hospital Materno Infantil de Brasília (HMIB) funciona como referência nesse tipo de atendimento. Assim que a mulher é acolhida no programa, ela é informada sobre três possibilidades diante da gravidez: realizar o abortamento, segundo as exigências legais; ficar com o bebê ou seguir com a gestação e entregar o bebê em adoção, que são as orientações da Norma Técnica do Ministério da Saúde de 2012, segundo Arrais e Zerbini (2020). Vale ressaltar que nem todas as mulheres que procuram o Programa realizarão o procedimento de abortamento. Seja porque a idade gestacional estava avançada, seja por um desejo dela de manter a gestação ou por não se enquadrar nos termos exigidos pela lei para interromper a gravidez, segundo preconizações da Norma Técnica sobre Abortamento humanizado (Brasil, 2011).

Em virtude do crescente número de mulheres que buscaram o programa, sejam pelas dificuldades em ter acesso a um serviço de saúde mental com esta especificidade e como também para apoiar de forma mais sistemática aquelas que pelas razões já apresentadas não interromperam a gestação, foi oferecido $\mathrm{o}$ atendimento psicológico nos moldes do Pré-Natal Psicológico (PNP) estabelecido por Arrais (2012, 2014 e 2020), adaptado às vítimas de violência sexual, com o intuito de favorecer a essas mulheres o suporte emocional necessário para que elas conseguissem passar pelo processo de gestação, parto, adoção ou maternidade.

Segundo estudo sobre o perfil das vítimas atendidas no PIGL, realizado por Arrais e Zerbini (2020), os dados do programa de 2013 a $2017,48,59 \%$ foi a porcentagem de mulheres que interromperam a gestação, ou seja, pouco mais da metade, não abortaram legalmente. Contudo, como já mencionado, foi oferecido o PNP para algumas dessas mulheres que 
demonstraram desejo de ter esse suporte da equipe do próprio programa.

Cabe aqui explicitar que este serviço psicológico não surgiu como uma forma de impedir, confundir ou evitar que a mulher aborte. Desde o primeiro atendimento, no acolhimento, são oferecidas a ela todas as informações segundo a legislação brasileira e as possíveis "opções" diante de uma gestação decorrente de estupro: interrupção, entrega para adoção e criação do bebê

Assim, nos anos de 2017 e 2018, após a negativa de abortamento, de acordo com a legislação vigente, e em virtude da necessidade e demanda psicológica, algumas mulheres foram atendidas no PIGL pelas psicólogas do programa, na modalidade de atendimento psicoeducativo e psicoprofilático, que se trata do (PNP). Foi uma demanda que surgiu ao longo dos anos de trabalho, pois, não havia lugares na Rede de Saúde Mental do Distrito Federal que pudessem acolher e tratar essas mulheres com esta especificidade, gravidez pós-estupro.

Nesta época, cinco pacientes, que não interromperam a gestação, começaram a ser atendidas com o PNP, independentemente do desfecho: ficar com o bebê, abortamento ou colocá-lo para adoção, contudo, nem todas deram continuidade aos atendimentos. A única paciente que aderiu e continuou o tratamento psicológico foi a mulher que participou da pesquisa de mestrado intitulada "Contribuições do Pré-natal Psicológico para proteção psíquica da díade mãe-bebê em caso de gestação decorrente de estupro: Um estudo de caso à luz da teoria da subjetividade" (Zerbini, 2020).

Buscou-se, no presente artigo, sistematizar a atuação psicológica nesses casos de gestação decorrente pós estupro. Partindo do acolhimento, abordando o desejo da mulher, oferecimento de todas as informações necessárias para que ela possa escolher o que fazer (abortar, entregar em adoção, ficar com o bebê) e caso ela decida seguir com a gestação um novo modelo de trabalho precisa ser oferecido, o PNP. Esse dispositivo pode ser utilizado individual ou em grupo, sendo o perfil das mulheres atendidas, mulheres que engravidaram após estupro e que desejam participar do grupo ou de sessões individuais com profissional da psicologia capacitado.

\section{Referencial teórico}

O Pré-Natal Psicológico (PNP) é uma prática complementar ao pré-natal ginecológico, voltado para maior humanização do processo gestacional e se propõe a prevenir situações adversas decorrentes desse processo (Arrais \& Araújo, 2016). O PNP é uma metodologia de promoção da saúde mental e de prevenção dos agravos psíquicos relacionados à gravidez, e maternidade, inclusive nos casos de gravidez decorrentes de violência sexual, segundo os estudos mais recentes de Arrais, Amorim e Rocha (2020), Carioca-Zerbini e Arrais (2020). É uma prática voltada para o atendimento psicológico das gestantes, a qual também estimula a integração de seus familiares nos cuidados desenvolvidos ao longo do ciclo gravídico puerperal. É a sistematização de um trabalho com um novo conceito em atendimento perinatal, voltado para maior humanização do ciclo gravídico-puerperal e a construção da parentalidade (Arrais, Amorim \& Rocha, 2020; Arrais \& Araújo, 2016).

$\mathrm{O}$ atendimento psicológico oferecido à gestante mostra-se importante para uma melhor compreensão da modificação de si, do meio, das relações e fortalecimento das capacidades do indivíduo para enfrentar situações novas relacionadas ao processo do gestar, parir e maternar. Maldonado (2017), estabelece que é na gravidez que se constroem os alicerces do vínculo entre a família e o bebê e quando começam a surgir as primeiras tensões, que, se abordadas nessa fase, tendem a ser mais rapidamente superadas, favorecendo o crescimento emocional e a formação de vínculos mais saudáveis na família.

A função do PNP é de ajudar a gestante a elaborar a situação presente, independentemente das possíveis experiências de desajustamento ou trauma no passado. A finalidade básica não é uma "cura" nem uma 
reestruturação profunda da personalidade, mas uma tentativa de melhorar o funcionamento presente e futuro por meio da elaboração da experiência imediata (Maldonado, 2017).

O PNP surgiu como uma técnica que possibilita a mulher gestante falar sobre suas questões psicológicas a respeito de suas novas configurações. Os aspectos psicológicos da gravidez sempre compareceram nesse momento de vida, contudo, a importância dada a esses aspectos é algo moderno. A dinâmica psíquica no processo do gestar vem sendo cada vez mais estudado e se percebeu que as percepções de dor, medo, tensão e competência atravessam a mulher e pode tornar seu processo mais ou menos difícil a medida do suporte emocional e informacional que ela recebe (Arrais et al., 2020; Arrais \& Araújo, 2016; Maldonado, 2017).

Estudos que relacionam a gestação decorrente de estupro e o acompanhamento psicológico da mulher gestante ainda são poucos na literatura, (Lordello, 2013; Lordello \& Costa, 2014; Onyango et al., 2016). Estes pesquisadores expuseram a relevância desses estudos e os benefícios de psicoterapias desde o mais cedo possível após a violência. Contudo, não foram encontrados outros estudos que tenham acompanhado o desenrolar da gestação e os anos seguintes.

Considerando a necessidade de mais estudos que tratem dessa temática, observou-se uma lacuna teórica e técnica importante. A proposta desse protocolo de intervenção é adaptar a metodologia do PNP e utilizá-lo como uma ferramenta útil de prevenção de adoecimentos psíquicos específico para vítimas gestantes em decorrência de estupro. Também visa, o PNP adaptado para essa clientela específica visar ser um potencializador para relação mãe-bebê saudável, caso a mulher decida seguir com a gestação e criar o bebê, ou que ela o entregasse para adoção, de forma a minimizar o impacto emocional que essas decisões possam lhes trazer a médio e longo prazo. Acentua-se aqui a importância de atendimento psicológico também para a mulher que vai interromper a gestação, visando minimizar os possíveis agravos psíquicos da violência que sofreu e ainda com relação ao abortamento, caso ela os apresente e deseje escuta psicoterapêutica.

O esperado é de que o PNP permita a mulher encontrar ambiente adequado para falar e elaborar seus traumas psíquicos e emoções relacionadas à violência sexual sofrida e que possa ressignificar a relação com o bebê que carrega em seu ventre e assim fazer a escolha necessária e possível.

No caso de uma mulher vítima de estupro que engravida, somam-se as angústias próprias de gerar um bebê com as angústias da vivência da violência, sua percepção social e pessoal a respeito de seu futuro e de sua escolha, aborto, adoção ou criação. O impacto do social, sobretudo da rede de apoio, sobre esta gestante tem fator primordial em seu enfrentamento e nas suas possibilidades de decisão (Onyango et al., 2016). Enfim, é importante conhecer e considerar o sentido subjetivo que ela dá para a vivência do estupro, da gravidez, do abortamento, do parto, da possibilidade de adoção, da maternidade e da relação mãe-bebê.

Reações de medo, tristeza, evitação social, vergonha, tristeza, percepção de estar suja, anedonia e ainda sintomas de TEPT são comuns nessas mulheres vítimas, antes e após o abortamento ou parto, quais sejam: pensamentos intrusivos sobre o trauma, pesadelos frequentes, revivência da cena traumática, apreensão (Silva \& Vagostello, 2017; Souza, Drezett, Meirelles, \& Ramos, 2013). Por isso a importância de um trabalho psicológico para ajudar esta mulher a elaborar esta situação traumática.

Esses achados científicos confirmam a importância do pré-natal psicológico como um suporte à mulher gestante e sua família para a prevenção de adoecimento psíquico durante o ciclo gravídico-puerperal. O PNP é, em si, um fator de proteção, pois oferece um espaço clínico, sigiloso e terapêutico, o que minimiza o impacto dos fatores de risco psicológico presente. Reconhecendo a necessidade desse 
tipo de assistência, casos de mulheres grávidas em decorrência de estupro, e que não puderam ou não quiseram interromper a gestação, conforme legislação vigente, poderiam se beneficiar desse tipo de acompanhamento. Não no sentido de fazê-las ficarem com os bebês, mas possibilitando acolher sentimentos como raiva, ódio, desespero e outros, em relação a si mesma, e consequentemente ao bebê que gestam. Esse amparo pode ser um apoio para que elas possam lidar com a gestação e decidir sobre seu futuro após o parto. Por isso, a assistência psicológica na gestação, por meio da utilização do PNP, parece ser um válido instrumento psicoprofilático que deve ser implementado como uma política pública em contextos da saúde (Arrais et al., 2020; Almeida \& Arrais, 2016).

Ter conhecimento sobre gestação, parto e puerpério é base para poder analisar as questões psicológicas inerentes a esse processo, visando unir os saberes sobre sintomas e reações orgânicas e as mudanças psíquicas que compõem o universo desse momento de vida da mulher e que geram impactos também em seu entorno.

A depressão pós-parto é uma das psicopatologias mais frequentes do ciclo gravídico-puerperal, sendo os fatores de risco para sua ocorrência eventos ou situações já estabelecidas que propiciam o surgimento de problemas emocionais após o parto (Golse, 2002). De modo geral, Schmidt, Piccoloto e Muller, (2005) agrupam os riscos para a DPP em três categorias: a primeira relaciona-se à qualidade dos relacionamentos interpessoais da mãe, particularmente com seu parceiro; a segunda refere-se à gravidez e ao parto e à ocorrência de eventos de vida estressantes; e a terceira relaciona-se às adversidades socioeconômicas. Acrescenta-se a gestação decorrente de estupro.

Portanto, podem ser incluídos como fatores de risco ou fatores predisponentes para DPP: ser primípara; ser mãe solo; conflitos e falta de apoio conjugal; evento de vida estressante, como perda de emprego ou morte de familiar; falta de apoio familiar e social, histórico pessoal ou familiar de doença psiquiátrica, mas principalmente a existência de episódios depressivos anteriores e durante a gravidez.

Fatores de proteção são condições associadas às influências que podem contribuir para uma melhor resposta a determinados eventos de riscos, tendo uma visão da interatividade dos fatores e o efeito produzido da experiência estressora ou protetora vivida pela pessoa, assim, estão associados aos recursos individuais que reduzem o efeito do risco.

Tostes (2012) apresenta que quando a assistência pré-natal proporciona, além do controle biológico, continente ou base consistente para a mulher e sua família, constitui-se em fator de coesão importante, pois viabiliza o cuidado obstétrico humanizado e integral. E pensando nesse cuidado podemos estendê-lo também para as questões psicológicas, pois, elas são influenciadas e influenciam estas outras questões orgânicas.

Ocupar-se de uma mulher puérpera para abordar as questões psicológicas do ciclo que envolve gestação, parto e puerpério, vai além do tradicional pré-natal com preparação para a chegada de uma nova vida. Uma vez que, é preciso inclusive levar em conta também o não desejo de gerar aquele ser e ainda todo ódio por aquela situação despertado, cujo resultado pode ser, inclusive, que a mulher possa escolher não ficar com aquele bebê. Reconhecer, e aceitar como profissional, que não existe esse despertar materno para todas as mulheres, e principalmente em casos em que, por meio de violência e grave ameaça, foram abusadas e que por infeliz consequência uma gravidez ocorreu.

Gestar não pode ser sinônimo de ser mãe e desejo de maternar, devendo se levar em consideração as inúmeras formas de a mulher lidar com isso, deixando claro e dando todas as informações e oportunidades para que ela aja segundo seu desejo neste momento, que seja, abortamento, adoção ou ficar com o bebê. Pois, para uma mulher já violada, não poder escolher o que fazer consigo com as parcas opções que 
lhe restam, é inconcebível que ela tenha que atender um imperativo social que não respeita seu querer e sua dignidade. Esta mulher tem o direito, garantido por lei, de escolher, e ela precisa contar com o apoio e suporte profissional para isso.

\section{Método}

Trata-se de uma proposta de intervenção relacionada à saúde mental no ciclo gravídico-puerperal, denominada pré-natal psicológico, que visa apresentar um modelo de trabalho para que as equipes de saúde, especialmente psicólogos perinatais, possam atuar nas dificuldades inerentes à gestação decorrente de estupro e minimizar os agravos psicológicos deste tipo de gestação. Foi desenvolvida no Hospital Materno Infantil de Brasília (HMIB), Distrito Federal como produto da dissertação intitulada "Contribuições do Pré-natal Psicológico para proteção psíquica da díade mãe-bebê em caso de gestação decorrente de estupro: Um estudo de caso à luz da teoria da subjetividade" (Zerbini, 2020), do Programa de Mestrado Profissional em Ciências da Saúde da ESCS/SES-DF. A referida pesquisa de mestrado foi aprovada pelo CEP/FEPECS, sob o $\mathrm{n}^{\mathrm{o}} 3.894 .660$ e registrada sob o CAAE $\mathrm{n}^{\circ}$ 2111.7319.5.0000.5553.
A pesquisa foi ancorada nos preceitos teórico-metodológicos qualitativos da Teoria da Subjetividade (González-Rey, 2005), e tratouse de um estudo de caso de uma mulher de 29 anos, solteira, na $23^{\mathrm{a}}$ semana de uma gestação decorrente de estupro, sobre a qual a gestante teve o seu pleito por aborto legal mal gerido, e foi obrigada a levar a gestação a termo. Ela iniciou um trabalho de Pré-Natal Psicológico (PNP) para que pudesse lidar com os agravos psíquicos do trauma, e com a escolha a ser feita após o nascimento do bebê, adoção ou tomá-lo como filho. No presente artigo, será apresentado a sistematização da adaptação do modelo Pré-Natal Psicológico, baseado no acompanhamento psicológico realizado com a participante da pesquisa durante a sua gestação, que poderá ser utilizado por profissionais de saúde, especialmente psicólogos perinatais, que trabalhem com vítimas de violência sexual.

\section{Resultados}

Será apresentado a seguir, a sistematização e o planejamento do PNP em casos de gestação pós estupro, que constituiu o produto final do Mestrado Profissional citado anteriormente.

Tabela 1

O Pré-Natal Psicológico em gestação decorrente de estupro: Protocolo de intervenção

\section{$\mathbf{1}^{\circ}$ Encontro}

Acolhimento e escuta da história da paciente: Acolhimento e escuta da história da paciente e da violência sexual ocorrida. Abordagem sobre as possibilidades

Temas geradores diante da gestação decorrente de estupro: abortamento legal (segundo a legislação), ou manutenção da gestação para entrega do bebê para adoção, ou para criação. Análise do estado mental e uso de escalas psicológicas.

Promover contato inicial, estabelecimento de vínculo de trabalho e discussão Objetivos das expectativas e dos medos em relação à decisão (abortamento, adoção ou ficar com o bebê).

\section{Recursos instrumentais}

Procedimentos
Setting terapêutico, escuta ativa. Escalas psicológicas.

1. Em sala reservada, a gestante é convidada a falar sobre si, toda a situação que a levou até ali e quais percepções ela tem sobre seus direitos e qual é seu desejo em relação à gestação.

2. Técnica de levantamento de sintomas e estressores para rastreio de TEPT, ideação suicida ou outra psicopatologia. 
3. Aplicação do instrumento de Completamento de Frases (González-Rey, 2005) e Escalas Beck.

4. Explicação sobre o trabalho psicológico e os seguimentos: preparação para abortamento, adoção ou ficar com o bebê.

5. Tarefa para casa: pedir que a mulher reflita e fale sobre sua decisão no próximo encontro.

\section{$2^{\circ}$ Encontro}

Temas geradores Ambivalências, decisão e ação.

Objetivos Levantamento sobre a situação da gestação (Idade gestacional e condições clínicas) e o desejo da mulher.

1. Avaliar sessão anterior e discutir a tarefa de casa.

2. Conhecer episódios significativos da semana.

3. Atualizar tempo de gestação.

4. Discussão das expectativas e medos em relação à escolha.

Procedimentos 5. Planejamento segundo a decisão da mulher. Caso seja o abortamento, acompanhá-la até o procedimento e após alta avaliar a paciente e encaminhar para serviço da rede de Saúde Mental, caso ela deseje a continuidade do tratamento psicológico.

6. Caso seja levar a gestação a termo seguir como PNP. Explicitação sobre o trabalho de PNP.

\section{$3^{\circ}$ Encontro}

Temas geradores A gestação decorrente de estupro e as mudanças no corpo e psiquismo.

Levantar angústias, ansiedades e tristezas relacionados à gestação e maternidade. Quais são as fantasias relacionadas ao ciclo-gravídico puerperal e

Objetivos sobre a "herança física e psíquica" do agressor/genitor. Refletir sobre as figuras parentais na gestação e pós nascimento no contexto de violência. Fornecer esclarecimentos adicionais sobre gestação.

1. Avaliação de rede de apoio. Quem oferece suporte emocional e social para a paciente.

Procedimentos 2. Promover reflexões sobre "adoção"; "Ser mãe do bebê que carrega no útero", destacando-se representações sociais e mitos da maternidade instintiva e perfeita, modelo patriarcal e características atuais da maternidade.

\section{$4^{\circ}$ Encontro}

Temas geradores Relação mãe-bebê e questões de transgeracionalidade.

Objetivos

Refletir sobre as mudanças ocorridas na maternidade de uma geração para outra.

Recursos

instrumentais

Observar a interação da gestante com o bebê intra-útero. Avaliar ambivalência.

Folhas e lápis de cor.

1. Pedir que as pacientes desenhem como eram quando bebês e de como elas imaginam as características dos bebês que gestam.

2. Avaliar as fantasias relacionadas ao bebê, a semelhança com o Procedimentos agressor/genitor e sua decisão de colocá-lo para adoção ou ficar com ele.

3. Em seguida, devem escrever frases ou palavras que representem como elas se enxergam após esta experiência e decisão.

4. Como elas lidam com o trauma da concepção e o desenrolar da gestação.

\section{$5^{\circ}$ Encontro}

Temas geradores Parto e decisão 


\author{
Objetivos \\ Recursos \\ instrumentais
}

Procedimentos
Ouvir a mulher em sua decisão e seu plano de parto, feito em outro encontro. Como ela deseja que seja conduzida a cena e quem ela quer que esteja com ela. Avaliar sintomas ansiosos ou angústias relacionadas ao parto.

Técnica de associação de ideias sobre seu desejo na hora do nascimento e os seguimentos com o bebê.

1. As gestantes devem escrever as cinco primeiras palavras/frases que associam com: parto, recém-nascido, maternidade, trauma, futuro.

2. Avaliar os principais medos e receios. E buscar estratégias para lidar com esses sentimentos.

3. Respeitar e apoiar a decisão tomada pela gestante.

4. Orientações sobre o trabalho de parto e principais orientações terapêuticas e mitos sobre distúrbios no puerpério.

5. Tarefa para casa: Refletir sobre os tipos de parto, e plano de parto. "Desejo ter contato pele a pele?". "Não quero contato com o bebê?”.

\section{$6^{\circ}$ Encontro}

Temas geradores Avaliação do trabalho e encerramento.

Objetivos

Autoavaliação da gestante. Avaliação do programa vivenciado. E despedida para o parto.

Recursos instrumentais

\section{Procedimentos}

\section{Escrever uma carta para si.}

1. Avaliar o processo do PNP e colocar pontos que foram trabalhados e quais dificuldades que ainda perduram.

2. Declarar a decisão segundo o plano de parto trabalhado.

3. Planos futuros são relatados (sobretudo no que concerne aos cuidados de si, dos filhos e das famílias).

4. O profissional se coloca a disposição para atendimento durante o trabalho de parto e parto caso seja desejo da paciente.

5. Encaminhamentos em casos de necessidade.

6. Agendamento de um encontro sete dias após o parto para avaliação de estado mental no puerpério.
Esta é uma sistematização básica para o atendimento psicológico nos moldes do PNP voltado para mulheres vítimas de estupro que engravidaram. $O$ número de sessões e a distância entre elas, pode ser avaliado a cada caso. Pode ser realizado tanto no âmbito da saúde pública quanto em serviços privados. Tendo em conta este cenário, entende-se que esse acompanhamento é imprescindível para que as gestantes desenvolvam estratégias destinadas ao enfrentamento dos estressores socioambientais e emocionais relacionados ao trauma, gestação, interrupção da gestação, parto, adoção e ter um bebê. Em síntese, o suporte socioemocional, informacional e instrucional fomentado pelo PNP pode contribuir para o bem-estar dessas mulheres, além de favorecer a avaliação de sintomas de ansiedade, depressão, TEPT e uma escolha tomada com mais recursos internos e externos.

Alerta-se, contudo, que este instrumento de forma alguma pode servir como impedidor do direito ao aborto segundo a legislação brasileira atual. Toda mulher, vítima de estupro, precisa saber desse direito e que possa ter todo suporte psicológico, médico e social para decidir se deseja interromper a gestação, seguir com a gravidez e entregar o bebê para adoção ou ficar com a criança para si. O PNP é uma sistematização de trabalho, não um dispositivo de controle. 


\section{Considerações finais}

Ao final desse trabalho de intervenção psicológica, foi possível abarcar um grande volume de conteúdos de sessões e instrumentos, o que nos ajudou compreender as produções subjetivas relacionadas ao processo de Gravidez pós-estupro e construção e sistematização do PNP específico para gestantes vítimas de estupro, que levou em consideração as especificidades da demanda, estupro, gestação, relação mãe-bebê e outras características próprias desse momento.

Ao longo da pesquisa foi confirmado o potencial protetivo do PNP para a vítima gestante, uma vez que, ele difere de concepções típicas de acompanhamento psicológico e com outros desafios que precisam ser trabalhados.

O PNP é um programa de baixo custo que pode ser desenvolvido tanto nos setores públicos quanto privado. Associando-se às práticas biomédicas de acompanhamento, possibilita um cuidado integral à mulher e ao seu bebê, prevenindo psicopatologias e promovendo saúde. E em casos em que o suporte psicológico se faz ainda mais necessário, é uma proposta de trabalho que permite minimizar os agravos decorrentes da gestação pós estupro. Por isso é de grande importância que as equipes de saúde conheçam esse tipo de trabalho psicológico, a legislação e busquem qualificação para atuarem nas dificuldades inerentes à gestação decorrente de estupro.

Torna-se útil pensarmos mais adiante e avaliar a necessidade de outras intervenções de acordo com as necessidades da díade, principalmente, no que tange à comunicação ou não da ação de concepção que gerou essa criança. Das limitações do trabalho, ele deve ser considerado apenas como uma proposta de instrumento que pode ser utilizado por equipes que trabalham com esta temática. Não devendo ser utilizado para dissuadir uma mulher de interromper a gestação ou forçá-la a ficar com o bebê que gesta. Outra limitação diz respeito às especificidades de cada mulher e grupo de mulheres que podem fazer parte dessa prática psicológica do pré-natal. Em razão da singularidade de cada uma, o instrumento pode ser adequado ou necessitar de mais encontros com outras temáticas. Por conta disso, fazemse necessários mais estudos e que tragam a experiência deste protocolo de intervenção para apontar suas falhas e acertos.

\section{Referências}

Almeida, N. M. C., \& Arrais, A. R. (2016). O Pré-Natal Psicológico como Programa de Prevenção à Depressão Pós-Parto. Psicologia: Ciência e Profissão, 36(4), 847-863. doi: 10.1590/1982-3703001382014

Arrais, A. R., \& Araujo, T. C. C. F. (2016). Pré-Natal Psicológico: perspectivas para atuação do psicólogo em Saúde Materna no Brasil. Revista da SBPH, 19(1), 103-116. Recuperado de

http://pepsic.bvsalud.org/scielo.php?script= sci arttext\&pid=S1516$08582016000100007 \& \operatorname{lng}=p t \& t \operatorname{lng}=p t$

Arrais, A. R., Cabral, D. S. R. \& Martins, M.

H. G. (2012). Grupo de pré-natal psicológico: avaliação de programa de intervenção junto a gestantes. Encontro: Revista de Psicologia, 15(22), 53-76.
Recuperado de

https://seer.pgsskroton.com/renc/article/vie $\underline{w} / 2480$

Arrais, A. R., Mourão, M. A., \& Fragalle, B. (2014). O pré-natal psicológico como programa de prevenção à depressão pósparto. Saúde e Sociedade, 23(1), 251-264. doi: https://doi.org/10.1590/S010412902014000100020

Arrais, A. R., Amorim, B., \& Rocha, L. (2020). Pré-natal psicológico na prática: da origem ao modelo Arrais. In N. A. Romagnolo, M. Benincasa, \& M. G. V. Heleno (orgs.), Maternidade, parentalidade e conjugalidade: estudos teóricos e empíricos em psicologia (pp. 53-78). Curitiba: CRV. 
Arrais, A., \& Zerbini, E. C. (2020). Estudo de caso de gestação decorrente de estupro: contribuições do pré e do pós-natal psicológico para proteção psíquica da díade mãe-bebê. In P. Staliano \& M. Mondardo (Orgs.), Violência contra a mulher: diálogos interdisciplinares. Curitiba: Brazil Publishing.

Brasil. Ministério da Saúde. (2011). Atenção humanizada ao abortamento: norma técnica (2. ed.). Brasília: Ministério da Saúde. Recuperado de https://bvsms.saude.gov.br/bvs/publicacoes/ atencao_humanizada_abortamento_norma_ tecnica_2ed.pdf

Golse, B. (2002). Depressão do bebê, depressão da mãe: conceito de psiquiatria perinatal. In L. Corrêa Filho, M. E. G. Corrêa, \& P. S. França (Orgs.), Novos olhares sobre a gestação e a criança até os 3 anos (pp. 232-248). Brasília, DF: LGE.

González-Rey, F. L. (2005). Pesquisa qualitativa e subjetividade: os processos de construção da informação (2. ed.). São Paulo: Cengage Learning.

Lordello, S. R. (2013). Menina, mulher, filha e mãe? A gravidez decorrente de violência sexual (Tese de doutorado). Instituto de Psicologia, Universidade de Brasília, Brasília, DF, Brasil.

Lordello, S. R., \& Costa, L. F. (2014). Gestação decorrente de violência sexual: um estudo de caso à luz do modelo bioecológico. Contextos Clínicos, 7(1), 94104. doi: $10.4013 /$ ctc. 2014.71.09

Maldonado, M. T. P. (2017). Psicologia da gravidez. Petrópolis: Vozes.

Onyango, M. A., Burkhardt, G., Scott, J., Rouhani, S., Haider, S., Greiner, A... Bartels, S. (2016). A Qualitative Analysis of Disclosure Patterns among Women with Sexual Violence-Related Pregnancies in Eastern Democratic Republic of Congo. PLOS ONE 11(10), e0164631. doi: 10.1371/journal.pone.0164631

Schmidt, E. B., Piccoloto, N. M., \& Muller, M. C. (2005). Depressão pós-parto: fatores de risco e repercussões no desenvolvimento infantil. Psico-USF, 10(1), 61-68. doi: 10.1590/S1413-82712005000100008

Silva, E., \& Vagostello, L. (2017). Intervenção psicológica em vítimas de estupro na cidade de São Paulo. Arquivos Brasileiros de Psicologia, 69(3), 183-198. Recuperado de

http://pepsic.bvsalud.org/scielo.php?script= sci_arttext\&pid=S1809$\underline{52672017000300013 \& \operatorname{lng}=p t \& t \operatorname{lng}=p t}$

Souza F. B. C., Drezett J., Meirelles A. C., Ramos D. G. (2012). Aspectos psicológicos de mulheres que sofrem violência sexual. Reprodução \& Climatério, 27(3), 98-103. Recuperado de https://www.elsevier.es/esrevista-reproducao-climaterio-385-articuloaspectos-psicologicos-mulheres-quesofrem-S141320871300006X

Tostes, N. A. (2012). Percepção de gestantes acerca da assistência pré-natal, seus sentimentos e expectativas quanto ao preparo para o parto (Dissertação de mestrado). Universidade de Brasília, Brasília, DF, Brasil.

Zerbini, E. M. C. (2020). Contribuições do pré-natal psicológico para proteção psíquica da díade mãe-bebê em caso de gestação decorrente de estupro: um estudo de caso à luz da teoria da subjetividade (Dissertação de mestrado). Escola Superior de Ciências da Saúde - ESCS, Brasília, DF, Brasil. 


\section{Dados sobre as autoras:}

- Elen Márcia Carioca Zerbini: Psicóloga da Secretaria de Estado de saúde do Distrito Federal. Especialista em Teoria Psicanalítica/UNB e Avaliação Psicológica/IPOG. Mestre em Qualidade na Assistência à saúde da mulher pela Escola Superior em Ciências da Saúde (ESCS). Psicanalista. Atendimento clínico a bebês, crianças pequenas e adultos. Avaliação e atendimento a bebês com riscos para o desenvolvimento psíquico. Psicóloga Perinatal.

- Alessandra da Rocha Arrais: Graduação (1992), Mestrado (1997), Doutorado (2005) e pósdoutorado (2017) em Psicologia Clínica e da saúde pela UnB. Psicóloga da Secretaria de Estado de Saúde do DF. Responsável técnica pelo serviço de psicologia do Hmib. Psicóloga clínica e perinatal da clínica Tons da Maternidade, em Brasília.

Declaração de Direito Autoral

A submissão de originais para este periódico implica na transferência, pelos autores, dos direitos de publicação impressa e digital. Os direitos autorais para os artigos publicados são do autor, com direitos do periódico sobre a primeira publicação. Os autores somente poderão utilizar os mesmos resultados em outras publicações indicando claramente este periódico como o meio da publicação original. Em virtude de sermos um periódico de acesso aberto, permite-se o uso gratuito dos artigos em aplicações educacionais e científicas desde que citada a fonte conforme a licença CC-BY da Creative Commons. 